\title{
Could localized warm areas inside cold caves reduce mortality of hibernating bats affected by white-nose syndrome?
}

\author{
Justin G Boyles $^{{ }^{*}}$ and Craig KR Willis ${ }^{2}$
}

White-nose syndrome (WNS) is a mysterious condition affecting populations of hibernating bats in the northeastern US. Little is known about its physiological effects on bats and no intervention has so far been proposed to slow associated mortality. We use an individual-based population model to examine how disruption of normal hibernation or pre-hibernation physiology could lead to the pattern of mortality that has been observed. We present evidence that artificial warming of localized areas within hibernacula could increase survival of WNS-affected bats during winter by lessening the energetic costs of periodic arousals. The model suggests that localized thermal refugia of $28^{\circ} \mathrm{C}$ could improve survival by up to $75 \%$, depending on how WNS acts to disrupt energy balance. This approach has yet to be tested, but it could serve as a stopgap measure to slow population declines until a solution is found, or to preserve remnant bat populations if WNS becomes ubiquitous throughout the ranges of affected species.

Front Ecol Environ 2010; 8(2): 92-98, doi: 10.1890/080187 (published online 5 Mar 2009)

Qince at least 2007, white-nose syndrome (WNS) has $\checkmark$ been decimating populations of hibernating bats in the northeastern US (Figure 1). The syndrome was first discovered in four caves in New York State in the winter of 2006/2007. By the winter of 2007/2008, it had spread to 29 additional caves, in five states within the region (Blehert et al. 2009), and already seems to have spread further this winter (2008/2009). The condition is named for its most obvious symptom, a psychrophilic (cold-loving) white fungus that grows on and in the skin of the face and flight membranes of bats. Affected individuals suffer severe weight loss and hundreds of emaciated bats have been found outside major hibernacula during winter (Figure 2), presumably searching for food (Blehert et al. 2009). Mortality estimates

\section{In a nutshell:}

- Hundreds of thousands of bats in the northeastern US have died as a result of white-nose syndrome (WNS) since the winter of 2006/2007

- WNS appears to be spreading and could lead to large-scale collapse of populations of common and endangered bat species, with far-reaching ecological consequences

- Providing localized, warm-temperature refugia within affected caves, while ensuring that overall cave temperature remains cold, could dramatically increase survival by reducing energy expenditure

${ }^{1}$ Center for North American Bat Research and Conservation, Department of Biology, Indiana State University, Terre Haute, IN *(jgboyles@ zoology.up.ac.za); ${ }^{2}$ Department of Biology and Centre for Forest Interdisciplinary Research, University of Winnipeg, Winnipeg, Canada

Beyond the Frontier: Listen to Craig Willis discussing this research on Frontiers' monthly podcast, at www.frontiersinecology.org. exceed $75 \%$ in affected sites, but in some large hibernacula, nearly $100 \%$ of the bats have been killed, and it is plausible that as many as half a million bats have already died (Hicks 2008). Many affected bats also suffer wing damage (Reichard 2008), which could reduce foraging success during the summer, leaving them more vulnerable if they enter hibernation in poor condition the following winter.

The WNS fungus has now been isolated and appears to be closely related to known species in the genus Geomyces (Blehert et al. 2009). There is no evidence that the fungus is transmissible to humans or to non-hibernating animals, as it appears unable to grow at temperatures above $20^{\circ} \mathrm{C}$ (Blehert et al. 2009). Its origins are unknown and, at present, there are no data that could help to determine whether the fungal outbreak is a natural occurrence or a human-caused phenomenon.

\section{The implications of WNS for ecosystems}

The greatest number of cases of WNS have been seen in the little brown bat (Myotis lucifugus), but individuals of at least five other species have been affected, including substantial numbers of IUCN-endangered (www.iucnredlist.org) Indiana bats ( $\mathrm{M}$ sodalis). These cave-hibernating species often congregate in large numbers at a few sites, so large proportions of populations could potentially be impacted. The long-term consequences of WNS are difficult to predict, but one distinct possibility is a full-scale collapse of populations of hibernating bat species in the northeastern US, and perhaps beyond. This is clearly of concern for management of bat populations, but could also lead to more general ecological consequences, in view of the role of insectivorous bats as the primary consumers of night-flying insects (eg Cleveland 
et al. 2006; Kalka et al. 2008). The ecological and economic importance of bats has not been well quantified in the affected region, but some "backof-envelope" calculations illustrate the point. Consider that an individual little brown bat generally consumes 4-8 $\mathrm{g}$ of insects per night during the summer (Anthony and Kunz 1977; Kurta et al. 1989; Encarnacao and Dietz 2006). Assuming that bats in the affected area hibernate for 200 days, this means that during the active season, each individual little brown bat could consume between 660-1320 $\mathrm{g}$ insects $\mathrm{yr}^{-1}$. Assuming that 500000 bats have died in the 2 years since WNS was first observed (Hicks 2008), this amounts to between 330 and 660 tonnes of annual insect biomass consumption, which has already been lost in the affected region. This impact will be compounded by the life histories of these bat species, characterized by long lifespans (occasionally exceeding 30 years in the wild) and low reproductive rates (only one or two young per year; Barclay and Harder 2003). If the disease spreads as expected, the ecological and economic impacts of WNS could extend well outside the northeastern US and will likely be prolonged.

\section{Energy balance in hibernating bats}

It is not known if the fungus is the direct cause of mortality or a symptom of a larger problem, but mounting evidence suggests that starvation during hibernation is the actual cause of death. Two competing - but not necessarily mutually exclusive - hypotheses have been proposed to explain the starvation. First, WNS may affect bats before the hibernation season begins, causing bats to arrive at hibernacula with insufficient fat to survive the winter, presumably because of some environmental stressor such as reduced food availability, altered insect community composition, or some unknown effect on summer feeding behavior. Alternatively, bats may arrive at hibernacula unaffected and with sufficient fat stores, but then become affected and use fat stores too quickly as a result of some disruption to hibernation physiology (WNS Science Strategy Group 2008).

All mammalian hibernators exhibit a characteristic pattern of body temperature $\left(T_{\mathrm{b}}\right)$ and metabolism throughout the winter that, roughly stated, consists of two distinct physiological states: long bouts of torpor and brief periodic arousals (Figure 3). A normal hibernator spends over 99\% of its time during winter in bouts of deep, prolonged torpor, during which $T_{\mathrm{b}}$ falls close to ambient temperature $\left(T_{\mathrm{a}}\right.$, $2-8^{\circ} \mathrm{C}$ for cave-hibernating bats) and metabolic rate can be reduced to less than $1 \%$ of that during euthermic (ie non- torpid) periods (Geiser 2004). However, for reasons that are still not fully understood, hibernators periodically arouse, at more or less regular intervals, to a normal $T_{b}$ and remain euthermic for periods lasting from less than a few hours to a day or more (Thomas et al. 1990; Geiser 2004). These periodic arousals make up less than $1 \%$ of the winter time budget, but are energetically expensive and can account for $80 \%$ or more of the total winter energy budget (Thomas et al. 1990). For bats in the cold hibernacula of the northeastern US, each periodic arousal requires costly thermogenesis (internal heat production) to warm $T_{\mathrm{b}}$ by as much as $35^{\circ} \mathrm{C}$ and then maintain it for up to several hours. The metabolic rate required to maintain a euthermic $T_{\mathrm{b}}$ at typical hibernaculum temperatures may be up to 400 times greater than that seen during torpor (Thomas et al. 1990). If WNS causes a disruption of this torpor-arousal cycle (ie by increasing the frequency or duration of arousals), it could easily cause bats to metabolize fat reserves too quickly, thereby leading to starvation. Indeed, one possibility is that skin irritation resulting from the highly invasive nature of the fungus (Blehert et al. 2009) might cause bats to remain out of torpor for longer than normal to groom, thereby exhausting their fat reserves prematurely.

\section{Modeling survival in WNS-affected populations}

We used an individual-based, bioenergetic model (IBM; Humphries et al. 2002; Boyles and Brack 2009) to examine how disruption in either torpor-arousal cycles or pre-hibernation fattening could lead to the pattern of mortality observed in WNS-affected bats. Briefly, the model creates individual bats with characteristics derived from published 

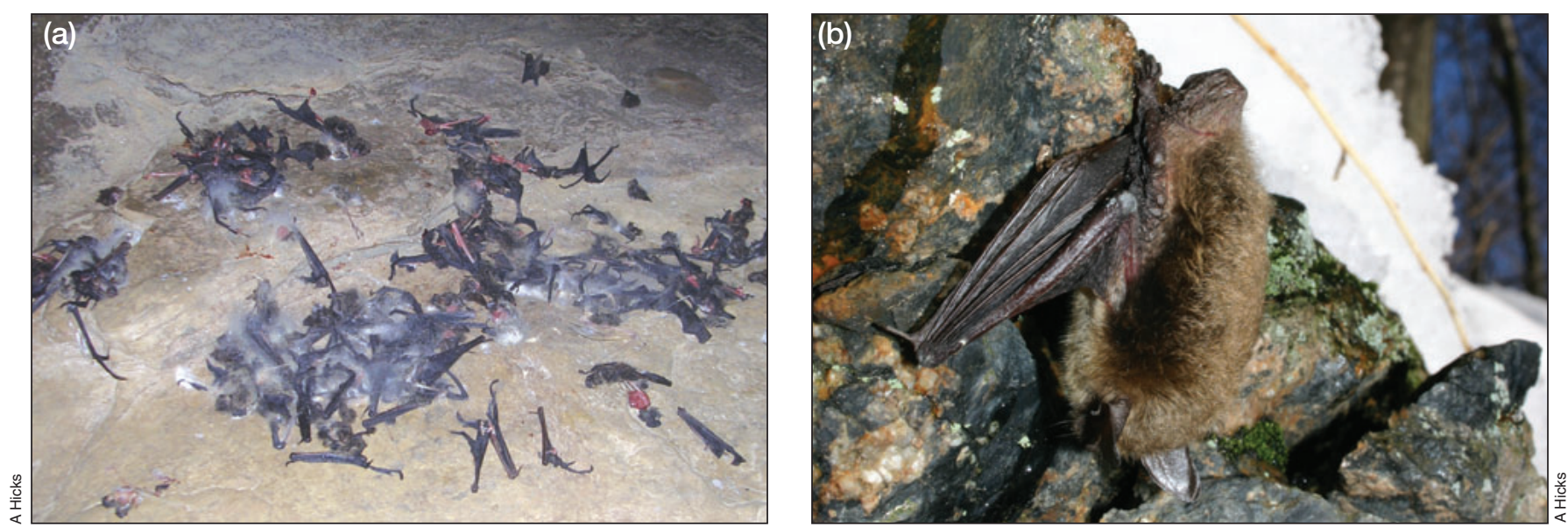

Figure 2. (a) Remains of bats on the floor of a hibernaculum affected by white-nose syndrome (WNS). Wings of some bats have presumably been removed and left by scavengers. (b) A little brown bat (M lucifugus) outside of a hibernaculum during daytime in winter - an unusual behavior, observed immediately before death in some bats affected by WNS. Note the snow in the background.

distributions of body mass, percentage body fat, arousal patterns, and other physiological and morphological traits (Brack and Twente 1985; Thomas et al. 1990; Johnson et al. 1998; Humphries et al. 2002, 2005). It then simulates individuals expending energy one hour at a time, throughout winter. During torpor, bats metabolize fat at the minimum torpid metabolic rate (TMR). During periodic arousals, they metabolize the amount of fat required to warm $T_{b}$ from $T_{a}$ to euthermic levels, remain euthermic for a given period at resting metabolic rate (RMR), then cool back into torpor at $67 \%$ of the cost of warming (Thomas et al. 1990). All bats are assumed to cluster during euthermic periods (Boyles et al. 2008), thereby reducing RMR by approximately $60 \%$ as compared with the costs of roosting individually (Canals et al. 1997). An individual survives hibernation if its initial fat mass is greater than that metabolized during winter. We assumed that hibernation lasted 200 days, beginning on 15 October, and that $T_{\mathrm{a}}$ was spatially and temporally stable at $2{ }^{\circ} \mathrm{C}$ throughout the hibernaculum. Boyles and Brack (2009) provide a full description of the model and its parameters.

To generate empirically testable predictions about physiological effects of WNS, we manipulated three model parameters for individual bats: the duration of torpor bouts (ie the frequency of arousals), the duration of periodic arousals, and the combination of pre-hibernation body mass and percent body fat. Body mass and body composition are biologically interdependent (Kunz et al. 1998), so this relationship is incorporated into the model and these parameters could not be altered individually (Boyles and Brack 2009). We manipulated these variables to recreate patterns of mortality recorded in bats found dead outside hibernacula and submitted to the New York State Department of Health for rabies testing in 2006/2007 (Figure 4). During that winter, the numbers of bats sent for rabies testing increased dramatically, beginning in January and peaking in February and March at more than 10 times the previous 25-year average, as WNS-affected bats were found near hibernacula (Blehert et al. 2009). WNS may also alter other parameters that could increase energy expenditure during winter (eg torpid or euthermic metabolic rate, propensity to cluster, or behavior during arousals) and presumably cause patterns of mortality similar to those observed in the affected region. We did not attempt to model these effects because of the absence of data with which to parameterize the model, but we stress that these effects should be investigated as more field and laboratory data become available.

The mortality curves (Figure 4) reveal that adjustments of two of the three parameters caused patterns of mortality that resemble those observed in New York State (ie approximately $75-80 \%$ mortality, with a peak in late February/early March): (1) a 600-hr reduction in the average duration of torpor bouts from $800 \pm 96$ hrs to $200 \pm 96 \mathrm{hrs}$ ( \pm 1 standard deviation); and (2) a 15-hr increase in the duration of arousals, from $3 \mathrm{hr}$ to $18 \mathrm{hr}$. The reduction in torpor bout duration necessarily results in an increase from approximately six arousals during winter (based on the natural arousal frequency estimated for hibernating little brown bats; Brack and Twente 1985) to 24 arousals. Likewise, the increase in arousal duration represents a $600 \%$ increase over that commonly assumed for little brown bats under normal conditions (Thomas et al. 1990; Boyles and Brack 2009). Changes in bout duration or bout frequency will both increase the time spent euthermic over the course of winter, but the energetic consequences of these adjustments are not equivalent because the reduction in torpor bout duration also increases the number of times each bat has to warm up and cool down, in addition to increasing the time spent euthermic.

Interestingly, we were unable to recreate the observed pattern of mortality by adjusting mean pre-hibernation body mass or percent body fat. Possibly due to the complex interrelationship between body mass and percent body fat (Kunz et al. 1998), nearly all bats in the model either died much earlier than the mid-winter peak of mortality or survived the entire winter (Figure 4). Although this suggests that disruption of the torpor-arousal cycle may be a more likely explanation for starvation than changes in pre-hibernation body mass or body composition, our modeling exer- 
cise is preliminary and should be interpreted cautiously, for three reasons. First, for simplicity we have only adjusted parameters in isolation and have not examined the effects of combined parameter adjustments, even though WNS may affect multiple parameters in combination. Second, so few data are available on hibernation physiology of little brown bats that values for some parameters in our model (eg TMR and arousal bout duration) are, by necessity, based on calculated estimates or data collected from captive bats in the laboratory. Hibernation physiology of free-ranging animals may differ in subtle but important ways. Third, we did not model other possible physiological or behavioral effects of WNS, such as elevated TMR or RMR, propensity for clustering, or activity levels during arousal. Nevertheless, the model does illustrate how disruption of torpor-arousal patterns could lead to the observed patterns of mortality. It also provides testable predictions that can be evaluated against empirical measurements as data on torpor patterns and bioenergetics of affected and unaffected bats become available. Importantly, the model can be readily modified as more data are collected.

\section{A possible solution to slow mortality associated with WNS?}

Until more is known about many aspects of WNS particularly whether or not the fungus is confirmed to meet the criteria of an infectious agent (ie Koch's postulates; Fredricks and Relman 1996) - it would be reckless to attempt an intervention that might prolong survival of affected bats in the wild. Increasing survival of affected animals could be disastrous if it increases the likelihood they will infect others and hastens the spread of the disease. However, it is still urgent that, at least on paper, we consider management strategies that could prolong survival of affected bats. Such approaches may prove critical if WNS is shown to be non-infectious, or if it becomes ubiquitous among North American populations, placing species at risk of extinction.

In the absence of a "cure" for WNS, there are only two management interventions that could prevent starvation in affected bats: (1) provide more energy to bats in the form of food or (2) reduce the amount of energy that bats must spend to survive hibernation. Providing bats with supplemental energy via a captive feeding/rehabilitation program would be extremely costly and time-consuming, even for small numbers of bats. Furthermore, given the time it takes to train bats to feed in captivity (Wilson 1988) and the fact that affected species probably do not normally feed during winter (Whitaker and Rissler 1993), provisioning thousands of free-living bats with supplemental food during hibernation is likely impractical. On the other hand, reduc-

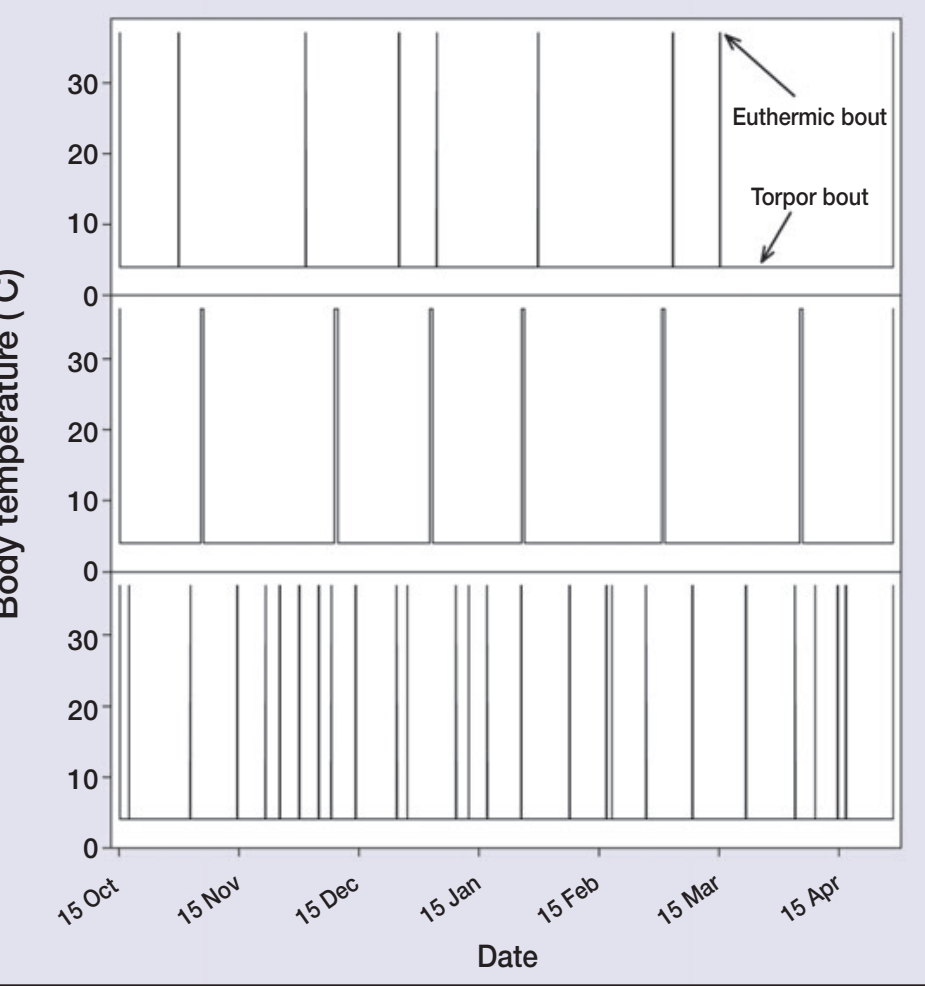

Figure 3. Example traces of body temperature $\left(\mathrm{T}_{b}\right)$ that are used by the model to determine individual survival and demonstrate effects of model parameter adjustments on hibernation patterns. (Top panel) Example of the "normal" hibernation pattern, based on data from the literature. (Middle panel) Representative hibernation pattern, assuming WNS causes bats to remain euthermic for $18 \mathrm{hrs}$ during periodic arousals (six times longer than normal). (Bottom panel) Example of a hibernation pattern assuming WNS causes bats to arouse, on average, every $200 \mathrm{hrs}$ (four times more often than normal). On average, bats remaining euthermic for $18 \mathrm{hrs}$ during periodic arousals are euthermic for a total of 108 hrs over the course of winter, whereas bats arousing every $200 \mathrm{hrs}$ are euthermic for $72 \mathrm{hrs}$ over the course of winter. However, the added warming and cooling costs associated with the extra arousals result in approximately equal energy costs and survival rates under the two scenarios if no thermal refugia are provided.

ing the energy expenditure side of the equation might be feasible on a large scale, with a much greater benefit and at lower cost.

We hypothesized that localized "thermal refugia" - areas of warm $T_{\mathrm{a}}$ inside hibernacula - could lessen heat loss during periodic arousals, thereby reducing energy expenditure enough to allow survival for substantial numbers of individuals. The efficacy of this intervention would depend on bats' ability to detect, and travel short distances to, these thermal refugia after arousing from torpor. However, in natural hibernacula with large thermal gradients, euthermic bats are often found in the warmest areas, presumably having flown there from cooler areas within the hibernaculum (Hardin and Hassell 1970). It therefore seems likely that bats would detect and exploit small patches of favorable microclimate if these were provided.

Introducing an artificial heat source of any kind into a bat hibernaculum is likely to be controversial, because it is 


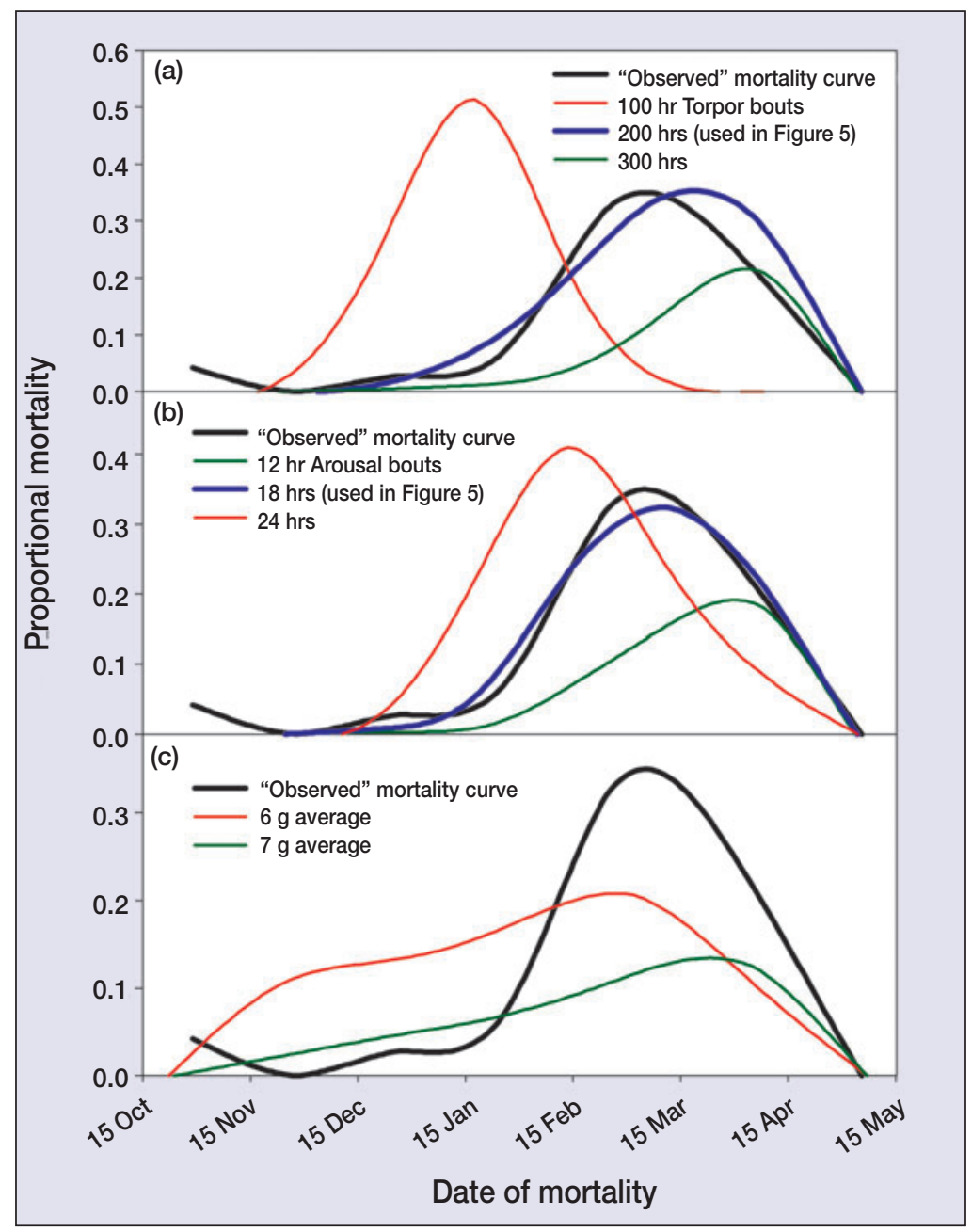

Figure 4. Mortality curves used to qualitatively determine model parameters that best simulate the mortality pattern observed in New York State in 2006/2007, extrapolated to 75\% mortality ("Observed" mortality curve). Mortality curves represent a smoothed curve taken from a 5-bin histogram of each dataset. (a) The effect of altering the duration of torpor bouts (ie the arousal frequency); (b) the effect of altering the duration of arousals; (c) the effect of changing body mass entering hibernation. In panels (a) and (b), simulation results that most closely matched predicted values are shown as thick blue lines. Thin lines represent simulation results based on parameter adjustments that do not qualitatively match the observed pattern of WNS mortality in New York. Panel (c) shows that adjustments of body condition did not lead to a pattern simulating observed mortality. In this simulation, a mean body mass of $6 \mathrm{~g}$ led to approximately the correct scope of mortality (74\%), but the wrong pattern. A mean body mass of $7 \mathrm{~g}$ led to approximately the correct pattern of mortality, but the wrong scope of mortality (40\%). Interestingly, no combination of adjustments for body mass and percent body fat improved the fit (see text).

widely held that cold temperatures are critical for successful hibernation. As long as $T_{\mathrm{a}}$ remains above the minimum setpoint for torpid $T_{\mathrm{b}}$ (perhaps $2-8^{\circ} \mathrm{C}$ ), cold temperatures do allow for the greatest energy savings during torpor (Humphries et al. 2002; Geiser 2004). Despite this, bats with large energy reserves often avoid hibernating at the coldest available $T_{a}$, because deep, prolonged torpor may be associated with physiological and ecological costs, and it is possible that warmer hibernaculum temperatures would benefit these individuals (Boyles et al. 2007). However, bats with relatively small reserves, including starving individuals affected by WNS, would probably require the coldest possible $T_{\mathrm{a}}$ to maximize energy savings during torpor. Therefore, under most circumstances, it would be essential that thermal refugia do not elevate overall temperature within a hibernaculum.

\section{Modeling the benefits of thermal refugia}

We used the IBM to quantify potential survival benefits of thermal refugia for WNS-affected bat populations. To do this, we first estimated mortality in an unaltered hibernaculum, where WNS-affected bats had to undergo periodic arousals at a $T_{\mathrm{a}}$ of $2^{\circ} \mathrm{C}$. We then modeled mortality in hibernacula where euthermic bats had the opportunity to exploit thermal refugia of $12^{\circ} \mathrm{C}, 20^{\circ} \mathrm{C}$, or $28^{\circ} \mathrm{C}$ after arousing from torpor. We chose $12^{\circ} \mathrm{C}$ to represent temperatures often used by euthermic bats in hibernacula south of the affected area, and $28^{\circ} \mathrm{C}$ because this value is near the lower critical temperature (LCT) of thermoneutrality for big brown bats (Willis et al. 2005); LCT for little brown bats is not known, but is probably similar.

In hibernacula provided with thermal refugia, we assumed that torpor bouts and warming and cooling phases of arousals occurred at a stable $T_{a}$ of $2^{\circ} \mathrm{C}$, but that euthermic periods occurred at thermal refuge temperatures of $12^{\circ} \mathrm{C}, 20^{\circ} \mathrm{C}$, or $28^{\circ} \mathrm{C}$. We did not incorporate flight costs to refuge sites, because these costs would be negligible over the short distances involved (ie less than $100 \mathrm{~m}$; Winter and von Helverson 1998). First, we ran survival simulations assuming an average torpor bout duration of $200 \mathrm{hrs}$ (ie an increase in arousal frequency over unaffected populations) and then repeated the simulations for populations exhibiting arousals of $18 \mathrm{hrs}$ duration (ie the two scenarios that led to the observed pattern of mortality in our simulations described above; Figure 4). We estimated survival in hypothetical populations of 1000 individuals and repeated simulations at each refuge temperature 100 times. We also used the model to determine the mass of fat remaining at the end of hibernation for survivors of single simulations of 1000 individuals at each refuge temperature. All simulations were run in Python 5.2.1 and statistical comparisons were done using Minitab 15.0.

In both scenarios, refuge temperature significantly affected survival (analysis of variance [ANOVA]: $P<0.0005$ ) and pairwise comparisons confirmed a significant increase in survival with each increase in refuge temperature (post-hoc Tukey's tests: $P<0.0005$ in all comparisons). If torpor bout 
length (ie arousal frequency) is the parameter affected by WNS, the model predicted average mortality in unaltered hibernacula of $81.9 \%$, which is consistent with the high rates observed in affected sites (Blehert et al. 2009). Mean mortality dropped to $63.0 \%$ in hibernacula with thermal refugia of $12^{\circ} \mathrm{C}$, $43.6 \%$ in hibernacula with refugia of $20^{\circ} \mathrm{C}$, and only $25.2 \%$ in hibernacula with refugia of $28^{\circ} \mathrm{C}$ (Figure $5 \mathrm{a}$ ). Thus, the model predicts a survival benefit of $57 \%$ if WNS is causing bats to increase their arousal frequency and they have access to $28^{\circ} \mathrm{C}$ thermal refugia.

The survival benefits were even more pronounced when duration of arousal periods was the variable influenced by WNS. In this scenario, mortality was $82.9 \%$ in unaltered hibernacula, dropping to $53.2 \%$ in hibernacula with thermal refugia of $12^{\circ} \mathrm{C}$, $25.5 \%$ in hibernacula with refugia of $20^{\circ} \mathrm{C}$, and only $7.5 \%$ in hibernacula with refugia of $28^{\circ} \mathrm{C}$ (Figure $5 \mathrm{c}$ ). The survival benefits (approximately 75\%) are so great in this scenario because bats spend about 35\% more time euthermic, which is when the benefit of thermal refugia would be realized, and because they undergo fewer episodes of warming and cooling, during which they gain no benefit from thermal refugia in the model. In either case, the reduced rate of mortality represents an enormous improvement for a disease that currently kills most individuals in an affected site.

The model also predicted that artificial thermal refugia would considerably improve the size of fat stores of surviving bats at the end of hibernation, regardless of which parameter is altered by WNS (ANOVA: $P<0.0005$; Figure $5 \mathrm{~b}$ and $\mathrm{d}$ ). Mean mass of remaining fat stores at the end of hibernation increased significantly with increasing refuge temperature in both scenarios as well (post-hoc Tukey's tests: $P<0.019$ in all comparisons). This is important because post-hibernation fat stores could be critical, especially for females, to fuel migration to summering grounds and allow for successful reproduction.

Both of the above scenarios are based on the assumption that WNS affects patterns of arousal during torpor. However, even if WNS affects hibernation without affecting arousal patterns as modeled here (eg by increasing metabolic rate during torpor), the thermal refuge approach could still provide a small but potentially important survival benefit. Reduced energy costs during arousals could still allow some individuals that would otherwise perish to fend off starvation during winter. As mentioned above, we were not able to model implications of other possible effects of WNS on physiology and behavior because of the absence of data. However, as additional measurements are collected from free-living and captive bats, it will be possible to refine our bioenergetic model to quantify these effects.

\section{Application of thermal refugia in affected hibernacula}

There is no question that the thermal refuge approach would be an unorthodox and possibly controversial management intervention. However, we argue that the unprecedented scope of this problem warrants consideration and testing of unorthodox approaches, particularly if there is evidence supporting their effectiveness. An urgent priority should be to test the hypothesis that bats would exploit thermal refugia during periodic arousals if these were available. Again, we caution that more data addressing a number of aspects of WNS are needed before thermal refugia (or any intervention to increase survival) can be tested in affected sites. Most critically, we need to know how WNS is contracted by bats and how it is being spread, because increasing survival rates could be disastrous if it increases the rate of spread of the disease. However, if WNS exhibits low rates of spread during the active season and overwinter survivors are unlikely to spread the disease during spring and summer, or if the disease becomes widespread and threatens species with extinction, thermal refugia could provide a critical "stop-gap" measure to preserve populations until a more permanent solution is found.

Equipment designed to warm refugia would need to be tailored to individual hibernacula, but we suggest that small heating units could be affixed in such a way as to warm domes or crevices in cave ceilings. These structures are used by euthermic bats during winter, probably because they trap metabolic heat and therefore reduce energetic costs during 
euthermic periods. Alternatively, small artificial structures, similar to the wooden "bat boxes" commonly occupied by bats during summer, could be used instead. Heaters would need to be regulated by thermostats to avoid exceeding the upper critical temperature of thermoneutrality (ie the $T_{\mathrm{a}}$ at which energy must be spent to lower $T_{b}$ back to acceptable levels) and, under most circumstances, it would be essential to avoid raising overall hibernaculum temperature. Humidity would also need to be monitored, because of the importance of high humidity for successful hibernation in bats. However, hibernacula are extremely humid environments and free-standing water or condensation is typically available, so humidity effects are unlikely. Use of refugia by bats could be quantified via passive integrated transponder (PIT) tags or infrared imaging observations of control and refuge sites. The efficacy of the technique in increasing survival could be measured directly or estimated indirectly from mass losses in altered versus unaltered hibernacula.

\section{Conclusions}

A continent-wide research effort has been mobilized to study the effects of WNS and determine if the fungus is the cause of mortality or a secondary symptom. However, while research is being conducted, it is very likely that bats will continue to die in unprecedented numbers. Techniques such as the computer simulations described here can play an important role in addressing questions about WNS that are either difficult or risky to address empirically, or will take longer to answer than is practical (Boyles and Brack 2009). Our modeling exercise also illustrates that computer simulations may be useful for testing the efficacy of possible management interventions. We have described the potential benefits of one such intervention, but the model could easily be modified to test other possibilities. The use of thermal refugia is limited in that it is a reactionary treatment of a symptom and is not likely to address the underlying cause of WNS. However, this approach might provide a logistically simple, verifiable method to reduce mortality until long-term solutions can be found.

\section{Acknowledgements}

We thank D Aubrey, FP Cryan, and J Karst for comments on the manuscript. JGB was supported by the Cave Conservancy Foundation Fellowship. CKRW's research is supported by the Natural Sciences and Engineering Research Council (Canada).

\section{References}

Anthony ELP and Kunz TH. 1977. Feeding strategies of the little brown bat, Myotis lucifugus, in southern New Hampshire. Ecology 58: 775-86.

Barclay RMR and Harder LD. 2003. Life histories of bats: life in the slow lane. In: Kunz TH and Fenton MB (Eds). Bat ecology. Chicago, IL: University of Chicago Press.

Blehert DS, Hicks AC, Behr M, et al. 2009. Bat white-nose syndrome: an emerging fungal pathogen? Science 323: 227.
Boyles JG and Brack Jr V. 2009. Modeling survival rates of hibernating mammals with individual-based models of energy expenditure. J Mammal 90: 9-16.

Boyles JG, Dunbar MB, Storm JJ, and Brack Jr V. 2007. Energy availability influences microclimate selection of hibernating bats. $J$ Exp Biol 210: 4345-50.

Boyles JG, Storm JJ, and Brack Jr V. 2008. Thermal benefits of clustering during hibernation: a field test of competing hypotheses on Myotis sodalis. Funct Ecol 22: 632-36.

Brack Jr V and Twente JW. 1985. The duration of the period of hibernation of three species of vespertilionid bats. I. Field studies. Can J Zool 63: 2952-54.

Canals M, Rosenmann M, and Bozinovic F. 1997. Geometrical aspects of the energetic effectiveness of huddling in small mammals. Acta Theriol (Warsz) 42: 321-28.

Cleveland CJ, Betke M, Federico P, et al. 2006. Economic value of pest control service provided by Brazilian free-tailed bats in south-central Texas. Front Ecol Environ 4: 238-43.

Encarnacao JA and Dietz M. 2006. Estimation of food intake and ingested energy in Daubenton's bats (Myotis daubentonii) during pregnancy and spermatogenesis. Eur J Wildl Res 52: 221-27.

Fredricks DN and Relman DA. 1996. Sequence-based identification of microbial pathogens: a reconsideration of Koch's postulates. Clin Microbiol Rev 9: 18-33.

Geiser F. 2004. Metabolic rate and body temperature reduction during hibernation and daily torpor. Annu Rev Physiol 66: 239-74.

Hardin JW and Hassell MD. 1970. Observations on waking periods and movements of Myotis sodalis during hibernation. J Mammal 51: 829-31.

Hicks A. 2008. White-nose syndrome: background and current status. www.caves.org/WNS/wns\%203-30-08\%20html/index.html. Viewed 12 Jan 2009.

Humphries MM, Speakman JR, and Thomas DW. 2005. Temperature, hibernation energetics, and the cave and continental distributions of little brown myotis. In: Zubaid A, McCracken GF, and Kunz T (Eds). Functional and evolutionary ecology of bats. Oxford, UK: Oxford University Press.

Humphries MM, Thomas DW, and Speakman JR. 2002. Climatemediated energetic constraints on the distribution of hibernating mammals. Nature 418: 313-16.

Johnson SA, Brack Jr V, and Rolley RE. 1998. Overwinter weight loss of Indiana bats (Myotis sodalis) from hibernacula subject to human visitation. Am Midl Nat 139: 255-61.

Kalka MB, Smith AR, and Kalko EKV. 2008. Bats limit arthropods and herbivory in a tropical forest. Science 320: 71.

Kunz TH, Wrazen JA, and Burnett CD. 1998. Changes in body mass and fat reserves in prehibernating little brown bats (Myotis lucifugus). Ecoscience 5: 8-17.

Kurta A, Bell GP, Nagy KA, and Kunz TH. 1989. Energetics of pregnancy and lactation in free-ranging little brown bats (Myotis lucifugus). Physiol Zool 62: 804-18.

Reichard JD. 2008. Wing-damage index used for characterizing wing condition of bats affected by white-nose syndrome. www.fws.gov/northeast/PDF/Reichard_Scarring\%20index\%20ba t\%20wings.pdf. Viewed 7 Nov 2008.

Thomas DW, Dorais M, and Bergeron J. 1990. Winter energy budgets and cost of arousals for hibernating little brown bats, Myotis lucifugus. J Mammal 71: 475-79.

Whitaker Jr JO and Rissler LJ. 1993. Do bats feed in winter? Am Midl Nat 129: 200-03.

Willis CKR, Lane JE, Liknes ET, et al. 2005. Thermal energetics of female big brown bats (Eptesicus fuscus). Can J Zool 83: 871-79.

Wilson DE. 1988. Maintaining bats for captive studies. In: Kunz TH (Ed). Ecological and behavioral methods for the study of bats. Washington, DC: Smithsonian Institution Press.

Winter Y and von Helverson O. 1998. The energy cost of flight: do small bats fly more cheaply than birds? J Comp Physiol B 168: 105-11.

WNS Science Strategy Group. 2008. Questions, observations, hypotheses, predictions, and research needs for addressing effects of white-nose syndrome (WNS) in hibernating bats. http://batcon.org/pdfs/WNSMtgRptFinal2.pdf. Viewed 7 Nov 2008. 